\title{
Solving Neutron Transport Equation in the Reactor using the Intergral Average Derivative Method
}

\author{
Phan Huy Thien \\ Department of Theoretical Physics, Faculty of Physics, Hanoi University of Science, Vietnam National University. \\ 334 Nguyen Trai street, Thanh Xuan, Hanoi, Vietnam
}

\begin{abstract}
Space and time dependent neutron diffusion equations with many energy groups and taking into account delayed neutrons are nonlinear partial differential equations. IAD method and finite difference method for simplified equations to partial differential equations often, then be rewritten in matrix form. General solution of differential equations contain the exponential matrix of matrix coefficients. Specific methods applied IAD (average method integral derivative) as an example to determine the flux reactor radius spherical $R$ reflector layer $R \notin-R$.
\end{abstract}

Keywords: The Neutron field in Reactor, Boltzman transport equation

\section{Introduction}

Neutron diffusion equation describes the density of neutrons in a nuclear reactor core. Many calculation methods have been proposed to solve diffuse neutron mostly based on the method of finite difference applied to the domain geometric shape such as in the case of two-dimensional $\mathrm{X}-\mathrm{Y}$ or R-Z. On the other hand with complex geometries using finite element method. In addition, the method of analysis [1], [2], the Monte Carlo method [4] the Boltzmann equation in the nuclear reactor has been developed with the complex calculation program. However, there are many difficulties in the process depending on how the calculation method applicable to each specific problem, they relate to the timing and geometry of the system should take into account. During the last few decades, some authors use the hybrid method between a method and a method of this group other groups to take advantage of these methods simultaneously overcoming the disadvantages and shortcomings of each method.

IAD method (average method integral derivative) by Phan Van Hap proposal [12] which we developed and perfected applied to solve the nuclear reactor in combination with traditional methods can solve the equation under a consistent algorithm including singularity where there is no common derivative. such as multi-step method, the method rather singular integrals with finite total was some authors to use and have good results in many problems - especially crack theory of mechanics of the continuous environment. Author J.J. Golecki has focused on the application method "phan van hap method" in a series of articles published in scientific journals from 1979 to 2007 [11].

In recent 6 decades, the calculation and design of nuclear reactors was a significant step forward, along with the development of the computer, we built a computational model based reactor facilities of nuclear reactors and review the method of calculation, the successes and limitations of previous methods, the proposed method of applying the average integral derivative (IAD method) to the solution of transport equation neutrons to describe the physical processes taking place in the nuclear reactor core. The result after the problems of nuclear reactors given matrix equation calculated neutron flux for each energy group.

\section{Diffusion Equation for Neutron and the IAD Method}

Based on the kinetic equation for the nuclear reactor neutron energy groups [5] after processing the input data is written

The IAD coordinated approach with some other methods

$$
\begin{aligned}
& \frac{1}{v_{g}} \frac{\partial}{\partial t} \Phi_{g}(\mathbf{r}, t)=\nabla \cdot D_{g}(\mathbf{r}) \nabla \Phi_{g}(\mathbf{r}, t)-\sum_{a_{g}} \Phi_{g}(\mathbf{r}, t)-\sum_{g^{\prime}>g}^{G} \sum_{s_{g, g^{\prime}}} \Phi_{g}(\mathbf{r}, t) \\
& +\sum_{g^{\prime}=1}^{G}\left(\chi_{g} v \Sigma_{f_{g^{\prime}}}(1-\beta)+\Sigma_{s_{g, g^{\prime}}}\right) \Phi_{g^{\prime}}(\mathbf{r}, t)+\sum_{i=1}^{I} \chi_{g, i} \lambda_{i} C_{i}(\mathbf{r}, t) ; g=1,2, \ldots, G
\end{aligned}
$$

$$
\frac{\partial}{\partial t} C_{i}(\mathbf{r}, t)=\beta_{i} \sum_{g=1}^{G} v \Sigma_{f_{g}} \Phi_{g}(\mathbf{r}, t)-\lambda_{i} C_{i}(\mathbf{r}, t)
$$

where $\mathbf{r}$ is the position vector $(\mathrm{cm}), t$ is the time $(\mathrm{s})$, $\mathrm{F}_{g}(\mathbf{r}, t)$ is the scalar neutron flux $\left(\mathrm{cm}^{-2} \mathrm{~s}^{-1}\right)$ in group $g$, $C_{i}(\mathbf{r}, t)$ is the concentration of delayed neutron precursors $\left(\mathrm{cm}^{3}\right)$ in group $i, v_{g}$ is the mean velocity of the neutron $\left(c m s^{-1}\right)$ in group $g, D_{g}(\mathbf{r})$ is the diffusion coefficient ( $\mathrm{cm}$ ) in group $\mathrm{g}, \mathrm{S}_{a_{q}}(\mathbf{r}, t)$ is the absorption cross-section $\left(\mathrm{cm}^{-1}\right)$ in group $g, \mathrm{~S}_{f_{g}}(\mathbf{r})$ is the fission cross-section $\left(\mathrm{cm}^{-1}\right)$ in group $\mathrm{g}, \mathrm{S}_{s_{g, g d}}(\mathbf{r})$ is the scattering cross-section 


\section{International Journal of Science and Research (IJSR) \\ ISSN (Online): 2319-7064}

Index Copernicus Value (2013): 6.14 | Impact Factor (2014): 5.611

$\left(\mathrm{cm}^{-1}\right)$ from group $\mathrm{g}^{\prime}$ to group $\mathrm{g}$ such that $\left(\mathrm{S}_{s_{g, g \phi}}(\mathbf{r})=0\right.$ khi $\left.g \phi>g\right), n$ is the mean number of fission neutrons, $c_{g}$ is the spectrum of prompt neutrons in group $g, c_{g, i}$ is the spectrum of $i$-group delayed neutrons in group $g, l_{i}$ is the decay constant $\left(s^{-1}\right)$ of group $i$ precursors, $b_{i}$ is the fraction of delayed neutrons in group $i$, and $b=\stackrel{\circ}{a}_{i=1}^{I} b_{i}$ is the total fraction of delayed neutrons.

Using the finite difference method and the IAD method [13], equation (1) and (2) leads to the following matrix:

$$
\begin{gathered}
\frac{d \Psi(t)}{d t}=\mathbf{A} \Psi(t)+\mathbf{B} \\
\Psi(t)=\left[\begin{array}{llllllll}
\bar{\Phi}_{1} & \bar{\Phi}_{2} & \ldots & \bar{\Phi}_{G} & C_{1} & C_{2} & \ldots & C_{I}
\end{array}\right]^{T}
\end{gathered}
$$

Here $\overline{\mathrm{F}}_{1}, \overline{\mathrm{F}}_{2}, \ldots, \overline{\mathrm{F}}_{G}$ is the averaged neutron flux in equations (1) and (2) using the IAD method:

$$
\lim _{\delta \rightarrow 0} \frac{1}{2 \delta} \int_{-\delta}^{\delta} \frac{\Phi_{i}\left(x_{0}+t h\right)-\Phi_{i}\left(x_{0}\right)}{t} d t=\bar{\Phi}_{i}\left(x_{0}, h\right) \equiv \bar{\Phi}_{i}
$$

and

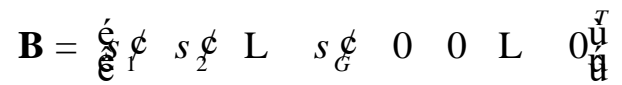

Here, $s_{g}, s \underset{g}{\notin} t_{g, g \oplus} f_{g, i}$ and $p_{i, g}$ was defined in reference [1]

Assume that, the matrices $\mathbf{A}$ and $\mathbf{B}$ are constant during the interval time $t_{m}$ and $t_{m+1}=t_{m}+h$ considering the length of the time interval is small. Then, the general analytical of equation (3) takes the following form:

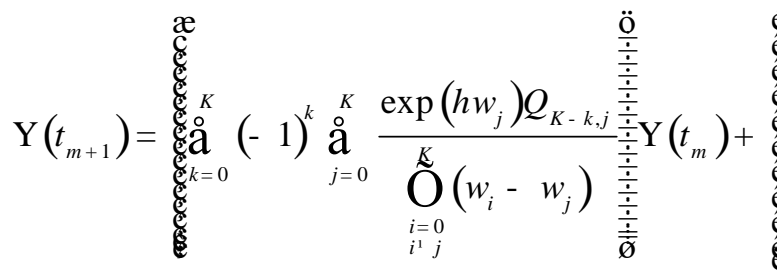

This equation is used in the numerical technique for solving the space-time neutron diffusion equations with multi-group of delayed neutrons which based on analytical method for the exponential function of matrix $\mathbf{A}$.

\section{Application to 2 Groups of Spherical Reactors}

We apply the IAD method to the specific problem of spherical reactor with two energy groups with a container region of radius $\mathrm{R}$ '- $\mathrm{R}$ (which acts as a reflect region) and a core of radius $\mathrm{R}$.. The diffusion equations for each groups can be written as:

$$
\begin{array}{ll}
\text { fast group } & -D_{1}^{C} \nabla^{2} \Phi_{1}^{C}(\mathbf{r})+\Sigma_{s}^{C} \Phi_{1}^{C}(\mathbf{r})=\frac{1}{k} v \Sigma_{f} \Phi_{2}^{C}(\mathbf{r}) \\
\text { thermal group } & -D_{2}^{C} \nabla^{2} \Phi_{2}^{C}(\mathbf{r})+\Sigma_{a}^{C} \Phi_{2}^{C}(\mathbf{r})=\Sigma_{s}^{C} \Phi_{1}^{C}(\mathbf{r})
\end{array}
$$

$\mathrm{Y}\left(t_{m+1}\right)=\exp (h \mathbf{A}) \mathrm{Y}\left(t_{m}\right)+(\exp (h \mathbf{A})-\mathbf{I}) \mathbf{A}^{-1} \mathbf{B}$

Expanding the expontial matrix $\exp (h \mathbf{A})$ using Taylor expansion:

$$
\exp (h \mathbf{A})=\stackrel{\circ}{\AA_{k}^{K}} a_{k}(h \mathbf{A})^{k}
$$

Where $a_{0}, a_{2}, \ldots, a_{K}$ are Taylor expansion coefficients and $K=G+I-1$ is the dimension of the matrixA.

The coefficient $a_{k}$ can be obtained by solving the system of equation:

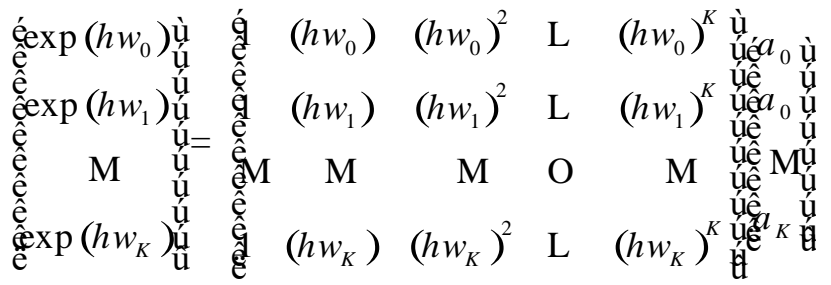

where $w_{0}, w_{1}, \ldots, w_{K}$ are the eigenvalues of the matrix $\mathbf{A}$. Gauss elimination method is used to solve the equation (9) which yields

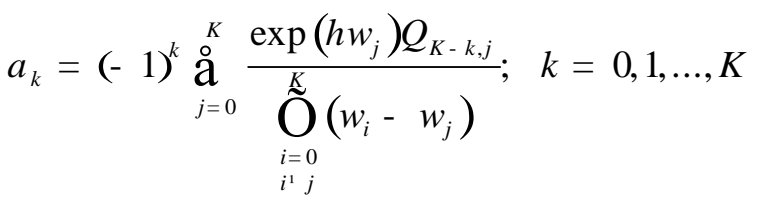

The coefficient $a_{k}$ can be expanded for two groups of neutron energies and for the mean group of delayed neutron. One gets:

constant $\mathrm{k}$ by solving the eigenvalue problem. The quantity $\Sigma_{s}^{C}$ is the cross section for scattering of the fast neutron to thermal neutrons and also plays the role of thermal neutron source; $\Sigma_{a}^{C}$ is the absorption cross section. The fluxes in the reflect region are described by

$$
\begin{array}{ll}
\text { fast group } & -D_{1}^{R} \nabla^{2} \Phi_{1}^{R}(\mathbf{r})+\Sigma_{s}^{R} \Phi_{1}^{R}(\mathbf{r})=0 \\
\text { thermal group } & -D_{2}^{R} \nabla^{2} \Phi_{2}^{R}(\mathbf{r})+\Sigma_{a}^{R} \Phi_{2}^{R}(\mathbf{r})=\Sigma_{s}^{R} \Phi_{1}^{R}(\mathbf{r})
\end{array}
$$

Here, $\Sigma_{s}^{R}$ is the scattering cross section from fast neutrons to thermal neutrons. Because there are no source of neutrons at the reflect region, therefore the right hand side of the first equation in (12) is zero. For the equation in the core region, the fluxes are the linear combination of different functions:

$$
\Phi_{1}^{C}(r) \equiv A_{1} Z(r)+C_{1} W(r) ; \Phi_{2}^{C}(r) \equiv A_{2} Z(r)+C_{2} W(r)
$$

The functions $Z$ and $W$ are determined from: 


\section{International Journal of Science and Research (IJSR) \\ ISSN (Online): 2319-7064}

Index Copernicus Value (2013): 6.14 | Impact Factor (2014): 5.611

$\nabla^{2} Z(r)+B_{1}^{2} Z(r)=0 ; \nabla^{2} Z(r)+B_{2}^{2} Z(r)=0$

where $B_{1}$ and $B_{2}$ are chosen such that the expressions

for $\Phi_{1}^{C}$ and $\Phi_{2}^{C}$ are solutions to equations (12)and

correspondingly. Using Eq. (15), one obtains

$$
\kappa_{3}^{2} \equiv \Sigma_{s}^{R} / D_{1}^{R} ; \quad \kappa_{4}^{2} \equiv \Sigma_{a}^{R} / D_{2}^{R}
$$

As a result, Eq. (13) becomes

$\nabla^{2} \Phi_{1}^{R}(r)-\kappa_{3}^{2} \Phi_{1}^{R}(r)=0 ; \quad \nabla^{2} \Phi_{2}^{R}(r)-\kappa_{4}^{2} \Phi_{2}^{R}(r)=-\frac{\sum_{s}^{R} \Phi_{1}^{R}(r)}{D_{2}^{R}}$

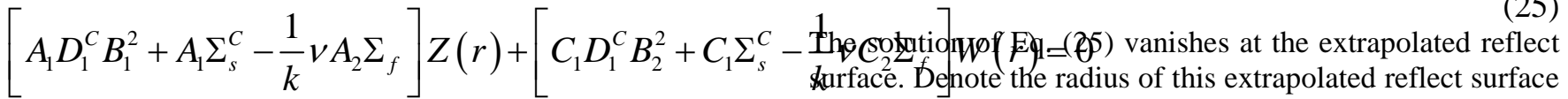

$$
\left[A_{2} D_{2}^{C} B_{1}^{2}+A_{2} \Sigma_{s}^{C}-A_{1} \Sigma_{s}^{C}\right] Z(r)+\left[C_{2} D_{2}^{C} B_{2}^{2}+C_{2} \Sigma_{a}^{C} \text { by } \tilde{R}_{1}^{\prime} \Sigma_{s}^{C}\right] W(r)=0
$$

Where $B_{1}^{2}$ and $B_{2}^{2}$ are solutions to:

$$
\left(B^{2}+\kappa_{1}^{2}\right)\left(B^{2}+\kappa_{2}^{2}\right)=\frac{k_{\infty}}{k} \kappa_{1}^{2} \kappa_{2}^{2}
$$

with the coefficients

$$
\kappa_{1}^{2} \equiv \frac{\Sigma_{s}^{C}}{D_{1}} ; \kappa_{2}^{2} \equiv \frac{\Sigma_{a}^{C}}{D_{2}} ; k_{\infty} \equiv \frac{v \Sigma_{f}}{\Sigma_{a}^{C}}
$$

The last expression is denoted by $k_{\infty}$ because is represents the coefficient for the case of infinite medium.. If expression (14) is a solution to the core equation (13) with Zand W given by (15). The quantities $B_{1}$ and $B_{2}$ are found from the equation (17):

$$
B^{2}=-\frac{\kappa_{1}^{2}+\kappa_{2}^{2}}{2} \pm \frac{1}{2} \sqrt{\left(\kappa_{1}^{2}+\kappa_{2}^{2}\right)^{2}+4 \kappa_{1}^{2} \kappa_{2}^{2}\left(\frac{k_{\infty}}{k}-1\right)}
$$

For a reactor, we have

$$
\frac{\sqrt{\left(\kappa_{1}^{2}+\kappa_{2}^{2}\right)^{2}+4 \kappa_{1}^{2} \kappa_{2}^{2}\left(\frac{k_{\infty}}{k}-1\right)}}{\kappa_{1}^{2}+\kappa_{2}^{2}}>1
$$

Assume $\mu^{2}$ and $\lambda^{2}$ are positive real parametersthe solutions of this quadtic equation are

$$
B_{1}^{2} \equiv \mu^{2} ; \quad B_{2}^{2} \equiv-\lambda^{2}
$$

Equation (15) becomes

$$
\nabla^{2} Z(r)+\mu^{2} Z(r)=0 ; \nabla^{2} Z(r)-\lambda^{2} Z(r)=0
$$

In the spherical coordinate system, the solutions are

$$
Z(r)=\frac{\sin \mu r}{r} ; \quad W(r)=\frac{\sinh \lambda r}{r}
$$

This set of equations has general solutions is the distribution of neutron fluxes in the reactor.. We convert those equation to the equation in the reflect region by introducing the quantities $\kappa_{3}^{2}$ and $\kappa_{4}^{2}$

$$
\Phi_{1}^{R}\left(\tilde{R}^{\prime}\right)=\Phi_{2}^{R}\left(\tilde{R}^{\prime}\right)=0
$$

(Z) $A_{1}+(W) C_{1}+(-U) S_{1}=0$

$$
\begin{aligned}
\left(-D_{1}^{C} Z^{\prime}\right) A_{1}+\left(-D_{1}^{C} W^{\prime}\right) C_{1}+\left(D_{1}^{R} U^{\prime}\right) S_{1} & =0 \\
\left(a_{1} Z\right) A_{1}+\left(a_{2} W\right) C_{1}+\left(a_{3} U\right) S_{1}+(-V) T_{1} & =0
\end{aligned}
$$

$\left(-a_{1} D_{2}^{C} Z^{\prime}\right) A_{1}+\left(-a_{2} D_{2}^{C} W^{\prime}\right) C_{1}+\left(-a_{3} D_{2}^{C} U^{\prime}\right) S_{1}+\left(-D_{2}^{R} V^{\prime}\right) T_{1}=0$

The symbols $Z, W, U, V$ and $Z^{\prime}, W^{\prime}, U^{\prime}, V^{\prime}$ are functions and their derivatives evaluated at $r=R$. Rewrite Eq.(27) in the matrix form

$$
\left[\begin{array}{cccc}
Z & W & -U & 0 \\
-D_{1}^{C} Z^{\prime} & -D_{1}^{C} W^{\prime} & D_{1}^{R} U^{\prime} & 0 \\
a_{1} Z & a_{2} W & a_{3} U & -V \\
-a_{1} D_{2}^{C} Z^{\prime} & -a_{2} D_{2}^{C} W^{\prime} & -a_{3} D_{2}^{R} U^{\prime} & D_{2}^{R} V^{\prime}
\end{array}\right]\left[\begin{array}{c}
A_{1} \\
C_{1} \\
T_{1} \\
S_{1}
\end{array}\right]=\left[\begin{array}{l}
0 \\
0 \\
0 \\
0
\end{array}\right]
$$

This system of linear equations (28) has non-trivial solution if and only if its determinant of the coefficients are zero

$$
\Delta(k) \equiv\left[\begin{array}{cccc}
Z & W & -U & 0 \\
-D_{1}^{C} Z^{\prime} & -D_{1}^{C} W^{\prime} & D_{1}^{R} U^{\prime} & 0 \\
a_{1} Z & a_{2} W & a_{3} U & -V \\
-a_{1} D_{2}^{C} Z^{\prime} & -a_{2} D_{2}^{C} W^{\prime} & -a_{3} D_{2}^{R} U^{\prime} & D_{2}^{R} V^{\prime}
\end{array}\right] \equiv 0
$$

This expression can be considered the threshold condition for the reactor in the two-group approximation because it is related to the parameters of the groups and the dimentions of the reactors. Determining the concentration of the nuclear fuel or the dimensions of the reactor allows one to determining other paramets of the reactor.

\section{Numerical Results}

To demonstrate the two-group model of the reactor, we solve the reactor equations numerical. The neutron multiplication factor of the model is given, and we adjust the fuel concentrations to satisfy the threshold condition (29). First, we calculate the radius of the reactor spherical core R, the radius of the extrapolated reflect surface, and points out some important characteristics of the reactor in our model:

\begin{tabular}{|l|l|l|l|l|}
\hline Core region & $D_{1}^{C}=1.44 \mathrm{~cm}$ & $D_{2}^{C}=0.229 \mathrm{~cm}$ & $\Sigma_{a}^{C}=0.1 \mathrm{~cm}^{-1}$ & $\Sigma_{f}=0.1 \mathrm{~cm}^{-1}$ \\
\hline Reflect region & $D_{1}^{R}=1.85 \mathrm{~cm}$ & $D_{2}^{R}=0.204 \mathrm{~cm}$ & $\Sigma_{a}^{R}=0.01226 \mathrm{~cm}^{-1}$ & \\
\hline
\end{tabular}

Using IAD method, and substitute the above parameters to Eq. (29), we obtain an algebraric equation for k. Solving this equation for the numerical value of the intersection of the horizontal axis with $\Delta(k)$ for $0 \leq k \leq k_{\infty}=2.5$. 


\section{International Journal of Science and Research (IJSR) \\ ISSN (Online): 2319-7064}

Index Copernicus Value (2013): 6.14 | Impact Factor (2014): 5.611

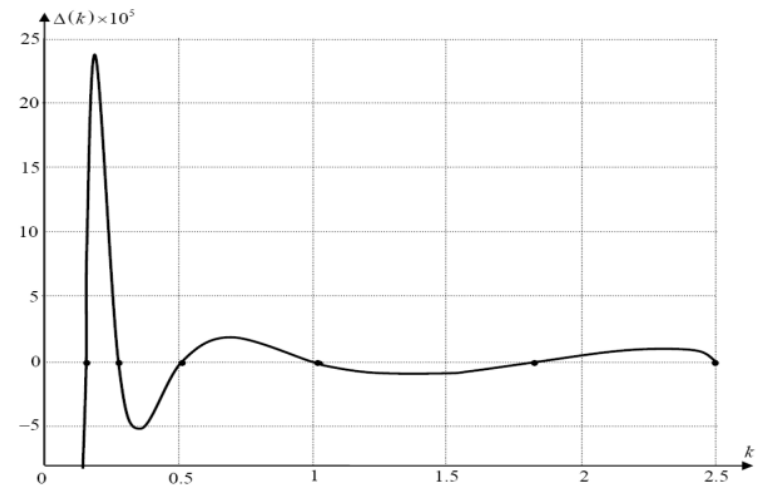

Figure 1: Solution of Eq. (29)

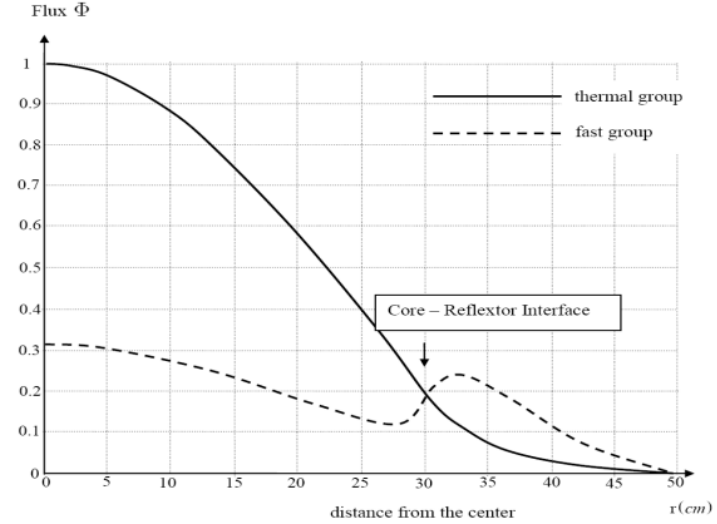

Figure 2: Flux of neutron in fast group and thermal group

The general approaches to one-group model can be easily expanded to the two-group model The consideration of twogroup model with a reflect region shows the flux of neutron in the reflect region which are not available in the one-group model. At the same time, the creation of a reflect region allows one to reduce the size of the reactor core as well as its mass. They provide valuable information when designing nuclear reactor as well as devices using nuclear energy.

\section{Conclusion}

Numerical calculation based on finite difference method for reactor with standard geometry is considered.. We use the IAD method to solve the neutron diffusion equation for complicated geometries. Combining with the finite difference method, we solve the diffusion equation for two energy groups and one group for delayed neutron.. The solutions are expanded for all points in the 3D mesh of the reactor geometry. They are compared to the traditional methods. It comes to our conclusion that the IAD method gives higher precision than other method because IAD method allows one to overcome the region or point of singularity.

\section{Acknowledgement}

The authors would like to thank our colleagues, Profs. Pham Ky Anh, Nguyen Suan Han, Phan Van Hap, Nguyen Quang Bau, for valuable discussions and comments. This work is supported financially by the grant from the Vietnam NAFOSTED agency, grant number 103.03 - 2012.2.

\section{References}

[1] W. M. Stacey, Nuclear Reactor Physics, JohnWiley \& Sons, New York, NY, USA, 2001.

[2] G. Arfken and H. Weber, Mathematical Methods for Physicists (5th edition, Academic Press, San Diego), 2000.

[3] K.S. Smith, An analytic nodal method for solving the two-group, multidimensional, static and transient neutron diffusion equations, M. Sc. Thesis, Nuclear Engineering, Massachusetts Institute of Technology, 1979.

[4] R.N. Blomquist and E.M. Gelbard, Alternative Implementations of the Monte Carlo Power Method, Nucl. Sci. Eng., 141, 85 (2003).

[5] D.L. Hetrick, Dynamics of Nuclear Reactors, American Nuclear Society, La Grange Park, 1993.

[6] E.G. Whitesides, Difficulty in Computing the k-Effective of the World, Trans. Am. Nucl. Soc., 14, 680 (1971).

[7] N.Z. Cho, et al., Refinement of the 2-D/1-D Fusion Method for 3-D Whole-Core Transport Calculation, Trans. Am. Nucl. Soc., 87, 417 (2002).

[8] A. Quarteroni, R. Sacco and F. Saleri, Numerical Mathematics, Springer-Verlag, New York, Inc. USA, 2000.

[9] S. Glasstone and A. Sesonske, Nuclear Reactor Engineering, Chapman \& Hall, 1994.

[10] N.Z. Cho, Fundamentals and Recent Developments of Reactor Physics Methods, Nuclear Engineering and Technology, 37, 25 (2005).

[11] Joseph J. Golecki, Direct displacement method in crack theory (numerical resolution), Meccanica, Volume 42, Issue 6, pp 555-566. 2007.

[12]Hoang Anh Tuan, Phan Huy Thien, Phan Van Hap. Advanced precision approximate solution of equations by means of continuous parameters. Proceedings of International Conference on Physics of nuclear reactor 19 th. Budapest, Hungary, 193 - 201, 1990.

[13] Phan Van Hap, Phan Huy Thien. The AD Method for the approximate solution of the Wigner Equations. Demonstratio Mathematica Vol XXVII, No 2. pp283286, 1994. 